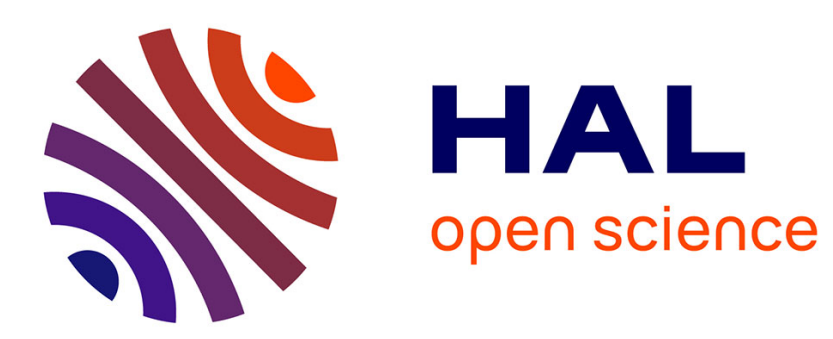

\title{
L'affaire des verriers de Givors ou le parcours du combattant des victimes de maladies professionnelles \\ Pascal Marichalar
}

\section{To cite this version:}

Pascal Marichalar. L'affaire des verriers de Givors ou le parcours du combattant des victimes de maladies professionnelles. Délibérée, 2019, №6 (1), pp.78-83. 10.3917/delib.006.0078 • halshs-02388806

\section{HAL Id: halshs-02388806 \\ https://shs.hal.science/halshs-02388806}

Submitted on 10 Dec 2019

HAL is a multi-disciplinary open access archive for the deposit and dissemination of scientific research documents, whether they are published or not. The documents may come from teaching and research institutions in France or abroad, or from public or private research centers.
L'archive ouverte pluridisciplinaire HAL, est destinée au dépôt et à la diffusion de documents scientifiques de niveau recherche, publiés ou non, émanant des établissements d'enseignement et de recherche français ou étrangers, des laboratoires publics ou privés. 


\title{
L'AFFAIRE DES VERRIERS DE GIVORS OU LE PARCOURS DU COMBATTANT DES VICTIMES DE MALADIES PROFESSIONNELLES
}

\author{
Pascal Marichalar
}

\author{
La Découverte | « Délibérée »
}

2019/1 N 6 | pages 78 à 83

ISSN 2555-6266

ISBN 9782348041785

\section{Article disponible en ligne à l'adresse :}

https://www.cairn.inforevue-deliberee-2019-1-page-78.htm

Distribution électronique Cairn.info pour La Découverte.

(C) La Découverte. Tous droits réservés pour tous pays.

La reproduction ou représentation de cet article, notamment par photocopie, n'est autorisée que dans les limites des conditions générales d'utilisation du site ou, le cas échéant, des conditions générales de la licence souscrite par votre établissement. Toute autre reproduction ou représentation, en tout ou partie, sous quelque forme et de quelque manière que ce soit, est interdite sauf accord préalable et écrit de l'éditeur, en dehors des cas prévus par la législation en vigueur en France. Il est précisé que son stockage dans une base de données est également interdit. 


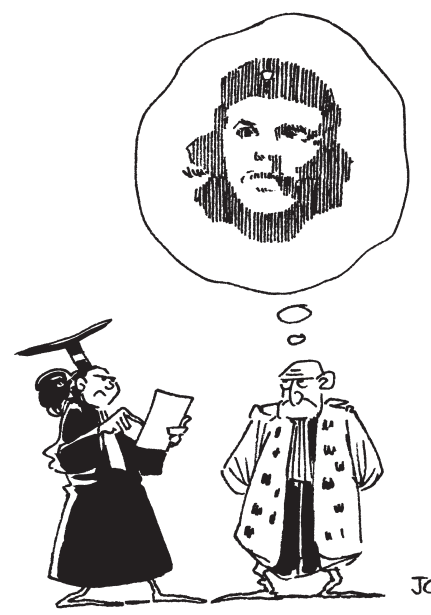

\section{GUÉRILLAS JURIDIQUES}

\section{L'affaire des verriers de Givors ou le parcours du combattant des victimes de maladies professionnelles par Pascal Marichalar}

En janvier 2003, la cheminée du four huit de la verrerie de Givors, établissement créé par un arrêté royal en 1749, a définitivement cessé de fumer. Avec cette fermeture, le groupe BSN, aussi connu sous le nom de Danone, s'est séparé de ses dernières filiales dédiées à l'emballage au terme d'une réorientation de plusieurs décennies, pour passer de la production du contenant à celle du contenu (eaux minérales, yaourts, etc.). Grâce à leur mobilisation pour amoindrir les terribles conséquences du plan social, les quelque trois cents salariés - principalement des ouvriers de sexe masculin - «laissés sur le carreau », selon l'expression qui nous vient du monde de la mine, ont pu bénéficier de reclassements ou, pour les plus âgés d'entre eux, de départs en préretraite.

Christian Cervantes était l'un des délégués CGT à la tête du mouvement contre la fermeture. À la fermeture de la verrerie, il a cinquante-cinq ans et peut prétendre à la préretraite. Il ne va pas en profiter longtemps. En 2005, il se voit diagnostiquer une tumeur cancéreuse à la gorge. En 2009, malgré les traitements et un espoir de rémission, une seconde tumeur est trouvée dans sa bouche. Son épouse, Mercedes Cervantes, aidée de leurs deux filles, va alors initier une enquête que l'on peut qualifier d' "épidémiologie populaire " $^{1}$ auprès des membres de l'association des anciens verriers, créée au lendemain de la fermeture.

1 Selon l'expression du sociologue Phil Brown dans son article «Popular Epidemiology and Toxic Waste Contamination: Lay and Professional Ways of Knowing », Journal of Health and Social Behavior, vol. 33, 1992, $n^{\circ} 3$, p. 267-281. 
Qui est malade? Qui est mort? Dans les 200 réponses, les Cervantes, aidés d'un militant local, Laurent Gonon, comptent pas moins de 92 cas de cancers et de nombreux cas inexpliqués de morts prématurées. La deuxième phase de l'enquête se focalise alors sur les conditions de travail dans la verrerie. À l'aide d'un travail d'archives, d'entretiens avec les anciens verriers et du dépouillement d'articles scientifiques (en collaboration avec des médecins et experts amis), Laurent Gonon dresse un tableau accablant des risques auxquels étaient quotidiennement exposés les salariés de la verrerie de Givors. Pas moins de dix produits pouvant être reconnus comme cancérogènes, en plus de situations de travail reconnues comme telles - ainsi, le travail posté en horaires alternés, « aux trois-huit ».

Qui savait? Toutes les mesures de prévention ont-elles été prises? L'enquête historique exhume la fiche de données de sécurité - notice rédigée par le producteur de produits chimiques dangereux - qui révèle, de manière cryptique et après dix pages d’affirmations lénifiantes, le terrible potentiel cancérogène d'une huile de graissage lorsque celle-ci est utilisée à la chaleur - ce qui, sur un moule de machine verrière qui reçoit des paraisons de verre à $800^{\circ} \mathrm{C}$, va de soi. Elle révèle des discussions en Comité d'hygiène, de sécurité et des conditions de travail (CHSCT) où les délégués syndicaux s'alarment de voir des produits dangereux encore utilisés malgré les promesses de substitution, ou déplorent que les masques qui leur sont offerts comme seule prévention sont inutilisables en conditions réelles de travail. Enfin, elle souligne la vanité du dernier combat d'un médecin du travail consciencieux pour que soit délivrée aux salariés licenciés l'attestation indiquant qu'ils ont été exposés à tant de poisons, afin qu’au moins ils puissent bénéficier d'un "suivi médical post-exposition »- combat perdu, par suite du refus du directeur de contresigner les attestations ${ }^{2}$.

\section{L'indemnisation comme seule justice}

À l'automne 2009, l'association des anciens verriers obtient de la mairie de Givors qu'elle organise une réunion avec les principales institutions concernées : l'administration sanitaire, le service de médecine du travail de la région, la préfecture et la direction de l'entreprise américaine qui a repris le passif du groupe verrier. Ces deux dernières n'envoient aucun représentant. Persuadés d'être les victimes d'un scandale sanitaire, les verriers demandent justice. Les personnes présentes leur décrivent l'unique voie vers la justice qui, selon eux, existe alors en France : lorsqu'une Caisse Primaire d'Assurance Maladie (CPAM) et/ou un employeur refusent de reconnaître le caractère professionnel d'une maladie, il faut alors demander cette reconnaissance, judiciairement, auprès du tribunal des affaires de sécurité sociale (TASS) ${ }^{3}$. C'est une procédure individuelle - en

2 Pascal Marichalar, Qui a tué les verriers de Givors? Une enquête de sciences sociales, Paris, La Découverte, 2017.

3 En se fondant sur l'article 461-1 du Code de sécurité sociale qui prévoit :

- si la maladie de la personne figure au tableau des maladies professionnelles de l'article R.461-3 du Code de sécurité sociale, la personne doit démontrer qu'elle est atteinte de cette maladie et que sa maladie a bien été contractée dans des conditions bien précises, spécifiées dans ce même tableau (délai de prise en charge, durée d'exposition ou liste des travaux effectués) ou, à défaut, quand l'une des conditions du tableau n'est pas remplie, elle doit démontrer par tout moyen que la maladie "est directement causée par le travail habituel de la victime »;

- si la maladie n'est pas désignée dans le tableau, son origine professionnelle peut être reconnue lorsqu'il est établi qu'elle est essentiellement et directement causée par le travail habituel de la victime et qu'elle entraîne le décès de celle-ci ou une incapacité permanente d'au moins $25 \%$. 
dépit de la similitude des conditions de travail et des pathologies, une maladie reconnue pour l'un des salariés ne facilite pas pour autant la reconnaissance de celle des collègues - qui est très exigeante, notamment en termes de documents et d'examens médicaux à fournir ainsi que d'étapes à franchir. Les démarches judiciaires initiées débouchant sur une reconnaissance du caractère professionnel d'une maladie sont donc très insuffisantes au regard du nombre de salariés malades de leur travail.

Outre ce problème du peu de maladies professionnelles reconnues comme telles, se pose la question des effets d'une telle reconnaissance: en France, la reconnaissance du caractère professionnel d'une maladie ouvre automatiquement pour le salarié le droit à une indemnisation financière forfaitaire de la part de la sécurité sociale (se répercutant, sous certaines conditions, sur les charges sociales des employeurs) mais sans véritable mécanisme de mise en cause de la responsabilité de l'employeur.

Ce système d'indemnisation "sans faute » - no-fault - n'est pas propre à la France. Il s'est diffusé à l'ensemble des pays européens au tournant du XXe siècle, ainsi que dans de nombreux États fédérés américains dans les années 1910 et 1920. Selon son principal thuriféraire contemporain, le philosophe devenu expert en assurances François Ewald, la loi française de 1898 sur l'indemnisation des accidents du travail aurait légitimé le passage d'une ère archaïque de la responsabilité à une ère moderne fondée sur l'assurance 4 . L'argument est tourné ainsi : aujourd'hui, dans notre "société du risque », il y aurait des risques inévitables, induits mécaniquement par l'essor industriel ; plutôt que de faire perdre du temps et de l'argent à coups de procédures en justice recherchant la responsabilité et la faute, il serait infiniment plus judicieux de socialiser le risque, en admettant une indemnisation forfaitaire sans conditions (ou presque...) des victimes. En retour de cette "automaticité » de la réparation, les victimes doivent évidemment renoncer à toute autre demande devant les tribunaux civils.

Comme dans bien d'autres affaires, les procédures initiées par les anciens verriers de Givors ont tôt fait de révéler l'envers de la médaille. Là où ils demandaient justice, on leur répond formulaire, certificat puis, dans le meilleur des cas, argent. Comme l'écrit la sociologue Anne Marchand, les victimes découvrent alors que "la mort par le travail semble socialement acceptée, puisque réduite à une formalité d'assurance dans le cadre d'une législation spécifique » ${ }^{5}$. Les «bons » cancers sont ceux qui correspondent aux critères des tableaux de maladies professionnelles. Les « mauvais » nécessitent des années de recours avant d'éventuellement aboutir. Les deux tumeurs de Christian Cervantes tombent dans cette dernière catégorie. La CPAM concernée avait ainsi refusé de reconnaître l'origine professionnelle de sa maladie. Finalement, ce n'est qu'en 2014 et en 2015 que deux décisions - l'une émanant d'une cour d'appel et l'autre de la Cour de cassation - ont abouti à la reconnaissance de l'origine professionnelle de la maladie de Christian Cervantes. Cependant, Christian Cervantes est mort depuis mars 2012. Ce sont ses proches qui ont

4 Michael Behrent, "Accidents Happen: François Ewald, the "Antirevolutionary" Foucault, and the Intellectual Politics of the French Welfare State », Journal of Modern History, 2010, p. 585-624.

5 Anne Marchand, "Quand les cancers du travail échappent à la reconnaissance », Sociétés contemporaines, n 102, 2016, p. 125. 
continué les procédures. Elles les poursuivent aujourd'hui, cherchant à faire appliquer judiciairement à la maladie professionnelle de Christian Cervantes le même traitement que celui obtenu dans les années 1990 à force de procédures judiciaires pour l'amiante : faire reconnaître que la maladie professionnelle dont a été atteint Christian Cervantes a été provoquée par une faute inexcusable de son employeur, telle que définie aujourd'hui par l'article L.452-1 du Code de la sécurité sociale. Cela implique de démontrer que l'employeur avait ou aurait dû avoir conscience du danger auquel était exposé le salarié et qu'il n’a pas pris les mesures nécessaires pour l'en préserver.

Au gré des batailles judiciaires menées par les avocats des victimes, la jurisprudence a progressivement complété la loi en précisant qu'il est indifférent que la faute inexcusable commise par l'employeur ait été la cause déterminante de l'accident survenu au salarié ou encore qu'il n'est pas nécessaire que le travail habituel soit la cause unique ou essentielle de la maladie. L'enjeu de la reconnaissance d'une faute inexcusable de l'employeur est éminemment symbolique, mais elle permet également d'obtenir des compensations financières plus importantes pour les salariés (ou leurs ayants droit).

\section{Un fossé entre droit pénal et épidémiologie}

Christian Cervantes fait partie de ces victimes de maladies professionnelles qui ne se sont pas contentées de reconnaissance symbolique et d'indemnisation. En 2009, affaibli par les cures et les opérations, il charge son avocate de déposer plainte contre X pour blessures involontaires. Dans les mois suivants, le procureur diligentait une enquête de police, dont les procès-verbaux ont pu être obtenus par l'association des anciens verriers. Médecin du travail, inspectrice du travail, responsable hygiène et sécurité, délégué syndical au CHSCT del'ancienne verrerie, ainsi que le président de l'entreprise repreneuse ont été convoqués au commissariat pour répondre à des questions souvent mal ficelées autour de l'exposition à des cancérogènes tels que «l'amiante » (sic). Était-il exposé à des conditions de travail dangereuses? Oui, bien sûr. Les mesures de prévention applicables étaient-elles toutes appliquées? Non. Pouvait-on prouver de manière certaine que les cancers de M. Cervantes ont été causés par son travail à la verrerie ? Non plus. Le juge d'instruction, prenant la suite de la procédure, diligentait une expertise médicale avec le même résultat. Deux éminents professeurs passèrent en revue la littérature médicale sur les cancers de $\mathrm{M}$. Cervantes et relevèrent bien une corrélation entre le type d'expositions qui faisait le quotidien d'un ouvrier de fabrication à la verrerie et les excès de ces types de pathologies. Pouvait-on pour autant en conclure que «s'il n'avait pas été exposé à ces conditions de travail pendant ses années à la verrerie, il n'aurait pas développé ces cancers » (la seule question qui intéressait le juge) ? Non, évidemment. Non-lieu.

La réponse était naturellement négative car la question était absurde. Dans l'état actuel de la science, le caractère cancérogène d'un produit est établi sur une base statistique : on relève que des populations exposées au produit présentent plus de cas de cancers que des populations non exposées. Dans certaines situations (grande entreprise, forte exposition, un seul agent causal), il est même possible de prédire, quasiment à l'unité près, l'excès de maladies qui sera causé par l'exposition à une substance. Pour autant, il n'est pas possible de savoir qui, parmi les personnes 
aujourd'hui malades de telle pathologie, ne l'aurait pas contractée si elle n'avait pas été exposée professionnellement.

L'inadéquation entre le droit pénal et la démarche épidémiologique qui fonde notre savoir sur la genèse des cancers : voilà la pierre sur laquelle achoppent toutes les plaintes pénales de victimes. En effet, les qualifications pénales les plus classiques pour poursuivre les responsables des catastrophes sanitaires sont des infractions dites "non intentionnelles », telles que l'homicide involontaire (prévue par l'article 221-6 du Code pénal) ou la mise en danger de la vie d'autrui (article 223-1 du Code pénal). Or l'existence de ces délits est très compliquée à caractériser, notamment parce que la loi du 10 juillet 2000, dite loi Fauchon, a fixé des exigences légales drastiques. En cas de lien indirect entre la faute et le dommage, le délit n'est en effet constitué que si a été commise une "violation manifestement délibérée d'une obligation particulière de prudence ou de sécurité » ou "une faute caractérisée exposant autrui à un risque d'une particulière gravité qu'il ne pouvait ignorer ». Étant donné ces conditions très restrictives, cette loi a donc entraîné une forme d'impunité des personnes en situation de responsabilité (dirigeants d'entreprise, élus, etc.) car les violations manifestement délibérées ou les fautes caractérisées sont très délicates à prouver, de même que leur lien avec le dommage survenu. Le traitement judiciaire de l'amiante illustre parfaitement cette lacune majeure de la loi pénale pour sanctionner les employeurs : la Cour de cassation, se heurtant au problème juridique de l'imputabilité, a récemment décidé de non-lieux dans plusieurs affaires autour de l'amiante parce qu'il n'était pas possible de déterminer si les victimes étaient tombées malades à cause des fibres d'amiante qu’elles avaient inhalées lorsque X était l'employeur (plutôt que son prédécesseur ou son successeur). Une recherche aussi insensée, d'un point de vue médical, que celle de retrouver la cigarette de trop qui aurait causé un cancer bronco-pulmonaire, et ce d'autant que le cancer résulte d'un ensemble d'expositions tout au long de la vie, qu’on résume parfois sous le nom d'exposome, plutôt que d'un événement isolé.

\section{De l'exposition intentionnelle à des poisons}

Du fait de cette inadéquation de la loi pénale - et du peu de volontarisme du législateur et de réflexion des juristes autour de cette question - il apparaît possible, dans notre pays comme dans bien d'autres, d'exposer des salarié·e·s à des poisons avérés sans courir le risque de poursuites pénales du moment que les maladies en question soient des cancers et surviennent après un certain délai de latence. Dans certains cas, il est possible d'établir fermement qu'il y a eu exposition, qu'il y a eu négligence et qu'il y a eu tel nombre de victimes (en excès de ce qui serait attendu). Mais comme il n'est pas possible d'identifier les victimes par leur nom, toute perspective de jugement pénal tombe à l'eau. C'est ce que le juriste italien Luca Masera dénonce comme un "renoncement à rendre la justice», dans une période où l'on se targue pourtant d'être plus attentif aux questions de santé professionnelle et environnementale ${ }^{6}$.

6 Luca Masera, «Évidence épidémiologique d’une augmentation de la mortalité et responsabilité pénale. À la recherche d'une qualification pénale pour une nouvelle catégorie épistémologique », Revue de sciences criminelles et droit comparé, 2015, p. 533. 
Il existe pourtant des moyens de contourner l'obstacle. S'inspirant des procès autour du thalidomide (médicament provoquant des malformations congénitales) dans l’Allemagne des années 1970, Masera s'est demandé ce qui, dans le droit pénal, oblige à identifier précisément la victime, au-delà de l'habitude de procéder ainsi. S'il est possible d'établir la chaîne causale entre le comportement du prévenu et la maladie ou le décès d'un nombre bien établi de personnes, n'est-ce pas suffisant pour juger? Dans le grand procès de l'amiante (groupe Eternit) à Turin entre 2009 et 2014, le Parquet de Turin (conduit par le procureur Raffaele Guariniello) avait développé une autre stratégie : poursuivre sur la seule exposition, en jouant notamment sur un article du Code pénal italien permettant de punir les propriétaires qui louaient des immeubles à la limite de l'effondrement - "et autres désastres intentionnels». Le simple fait d'exposer les salarié·ess à l'amiante avait été reconnu comme une infraction en soi. La survenue des maladies, établie épidémiologiquement, n'avait fait que confirmer la survenue du désastre et aggraver la peine. Ces décisions judiciaires historiques ont néanmoins été infirmées en dernière instance par la Corte di cassazione, sans renvoi devant de nouvelles juridictions, pour cause de prescription.

Enfin, au-delà des difficultés à sanctionner des comportements non intentionnels, se pose également la question de la prise en compte de l'intention lorsqu'elle existe. Dans l'affaire des verriers de Givors, si les employeurs n’ont généralement pas prémédité de tuer leurs salarié·e·s, il semble peu adapté de les poursuivre pour des infractions non intentionnelles, comme s'il sagissait d'un accident de la route causé par les seules intempéries. Car il semble bien que des décisions aient été prises intentionnellement de poursuivre des procédés qui exposaient les travailleurs à des produits toxiques. Il s'agirait alors de rester sur le terrain des infractions intentionnelles mais en développant le recours à la notion de «dol éventuel » pour caractériser l'intention des employeurs. Le "dol éventuel» peut se définir comme une volonté de nuire reposant sur une prise de risque, c'est-à-dire que l'auteur a conscience d'un possible résultat dommageable de son action, mais il agit néanmoins ou ne fait pas ce qui est en son pouvoir pour l'éviter ou en atténuer les conséquences. Dans l'affaire de l'incendie d'une usine sidérurgique qui avait fait sept morts à Turin fin 2006, le Parquet du Turin avait d'ailleurs choisi de retenir la qualification d'homicide intentionnel, en faisant valoir le «dol éventuel » du responsable des conditions de travail. Comme le montraient des mails saisis lors de perquisitions, il avait été alerté préalablement sur le risque d'incendie sur une ligne, et avait choisi de ne pas investir dans la sécurité tout en connaissant les potentielles conséquences. Le capitalisme contemporain est fait de telles décisions, qui conduisent à ce que l'avocat Jean-Paul Teissonnière appelle des «crimes d'indifférence ». 